\title{
Things I wish I had been taught about rhinoplasty
}

Tove performing rhinoplasties. They have the complexity of chess, and high stakes for the patient and surgeon. But I wish I did not have to learn that the hard way.

If I were teaching rhinoplasty to keen young plastic surgeons, these are a few concepts to begin with that might make the operation better:

1. The more mature the patient, the better the result. Measure maturity in accomplishment, not in age.

2. This can be a two-stage procedure. Say so at the start. If you don't need the second stage, you're lucky.

3. Perform the first stage under general anesthesia, the second under local anesthesia and sedation after you've seen how the patient handles the first operation. The final result takes one year. Say so. There is a cost for the second stage.

4. Women of all ages know what they want their nose to look like. Teens will always have trouble expressing this because they lack the vocabulary, but they know. They change their minds often, so you need to make sure that the goal is clear and decided on, with their parents too. Men seeking rhinoplasty know what they want less often. When you solve the difference in this riddle, you will better understand the psychology of rhinoplasty.

5. External rhinoplasty involves making a flap. If the skin is thick, edema will be long lasting. Flaps sometimes die. Use what you know about flaps to perform an external rhinoplasty judicially. The patient may be only able to 'take' one external rhinoplasty in a lifetime.

6. The key to rhinoplasty is the skin (well, cartilage too). Classify skin in the upper half and lower half of the nose. Think very thin, thin, medium, thick and very thick skin. You can't change this, you have to accept it. Very thin skin means every little bump will be felt and sometimes seen, so take time to smooth all the irregularities. Sculpt! Very thick skin in the nasal tip means you should never perform a rhinoplasty. No matter what you do to the cartilage, the tip will be bigger after surgery, not smaller! Try explaining that to the patient after surgery!

7. Perform a very good preoperative assessment with sketches, showing what you want to do and, most importantly, why you want to do it. This will remind you to stick to your game plan at surgery.

8. 'Give as good as you get'. Augment and reduce. Don't leave augmentation to secondary surgery: augment inadequate projection primarily.

9. Master the reproducible atraumatic infracture.
10. Master local anesthesia for rhinoplasty. It works well and is safe, but takes time to take effect. Performing it well is almost as complex as performing the operation itself.

11 . Don't routinely separate the upper lateral cartilages from the septum unless the nose is very wide in the middle third (rare).

12. Do learn how to use spreader grafts to widen the middle third of the nose, and how to make the nose look less deviated.

13. Don't be upset when your patient with the best result comes back and wants to be even better. Take it as a compliment.

14. Delicate technique means a better result. Eliminate everything indelicate, instituting fine instruments and fine procedures that work.

15. Master internal rhinoplasty. The recovery is much faster, and you can see everything you need to see.

16. Learn how to say 'no'. "I do not have the skill to achieve what you want", is a good way. Say it on the first visit.

17. Look for the tips with inadequate projection. Augment tips remembering that patients believe that the three-quarter view is the profile.

18. Time spent in preoperative analysis is never wasted; indeed, it is one key to a good result.

19. Lots of lateral crurae are almost vertical. Don't amputate the lateral part thinking the lateral crurae are transverse.

20. Look for cartilaginous nasal bones. Leave them untouched.

These are just a few things that might help in performing rhinoplasties.

There are many more. Experience means discovering them.

John R Taylor 